\title{
Swiss Science Concentrates
}

\section{A CHIMIA Column}

[(Cp-R)M(CO) $)_{3}$ (M = Re or ${ }^{99 \mathrm{~m} T c)}$ Arylsulfonamide, Arylsulfamide, and Arylsulfamate Conjugates for Selective Targeting of Human Carbonic Anyhdrase IX

D. Can, B. Spingler, P. Schmutz, F. Mendes, P. Raposinho, C. Fernandes, F. Carta, A. Innocenti, I. Santos, C. T. Supuran, and R. Alberto*, Angew. Chem. Int. Ed. 2012, 51, 3354.

Universities of Zürich, Florence (I) and Instituto Technologico e Nuclear, Sacavém (P)

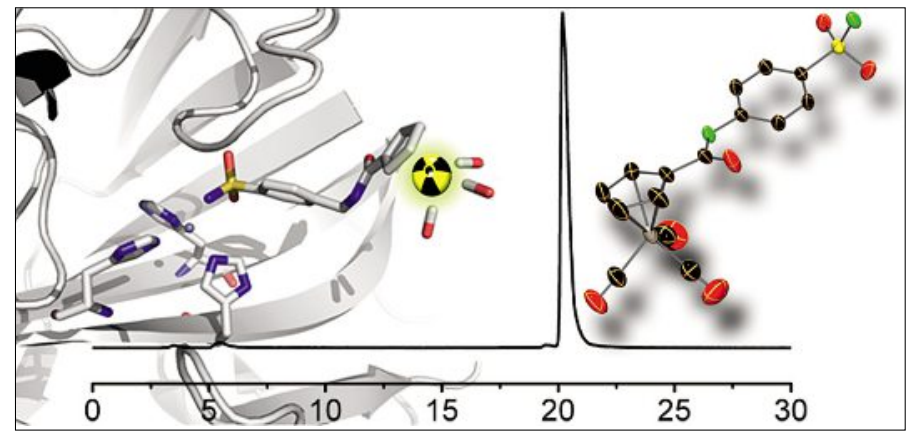

Human carbonic anhydrases (hCA) are important enzymes for which certain isoforms (e.g. hCA IX, hCA XII) are overexpressed in many diseases including cancer. Inert $\mathrm{Cp}$-based piano-stool complexes offer versatile scaffolds as hCA inhibitors. The authors have identified highly specific nanomolar-range inhibitors for hCA IX, XII and XIV. Upon combining the radioactive Cp ${ }^{99 \mathrm{~m}} \mathrm{Tc}$ complex with its non-radioactive homologue, Re, both imaging and therapy (i.e. theragnostics) can be achieved with a similar scaffold.

\section{Atropisomeric (P,N) Ligands for the Highly Enantioselective Pd-Catalyzed Intramolecular Asymmetric $\alpha$-Arylation of $\alpha$-Branched Aldehydes}

P. Nareddy, L. Mantilli, L. Guénée, and C. Mazet*, Angew. Chem. Int. Ed. 2012, 51, 3826.

University of Geneva

The development of chiral ligands for asymmetric transformations is a thriving discipline with unbroken momentum despite the wealth of published ligand structures. It gains its appeal not only from the creative basis of the process, but especially from the

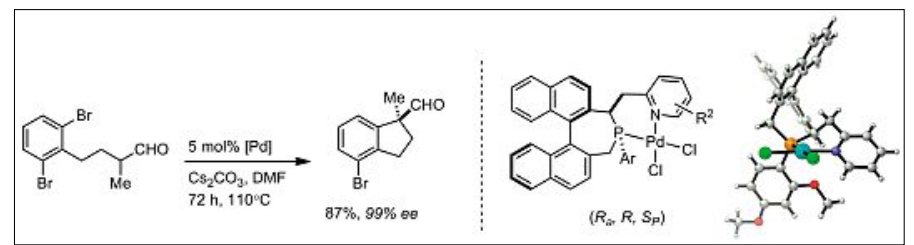

accomplishments in an ever-growing number of enantioselective catalytic reactions, considered to be particularly challenging. A concise synthetic route to a versatile ligand motif incorporating three elements of chirality is presented. The ligands employed in the title reaction lead to quarternary stereocenters with very good enantioselectivities. A dibrominated substrate was converted in high yield and with excellent $e e$, suggesting that, remarkably, reversible oxidative addition can take place.

\section{Total Synthesis of the Bacterial RNA Polymerase Inhibitor Ripostatin B}

F. Glaus and K.-H. Altmann*, Angew. Chem. Int. Ed. 2012, 51, 3405.

ETH Zürich

Given the increasing number of drug-resistant bacteria, the search for potent antibiotics with a new mode of action is crucial. Ripostatins A and B are macrolides that display a fundamentally different inhibition mode for bacterial RNA polymerase compared to well-established rifamycin-type inhibitors. To generate the chemical data for structure-activity relationship studies (SAR) with ripostatins, the authors report a modular and highly stereoselective total synthesis of ripostatin B. The target molecule was obtained in $3.6 \%$ overall yield over 21 steps in the longest linear sequence. A ring-closing metathesis was the key step to afford the fourteen-membered macrolide.

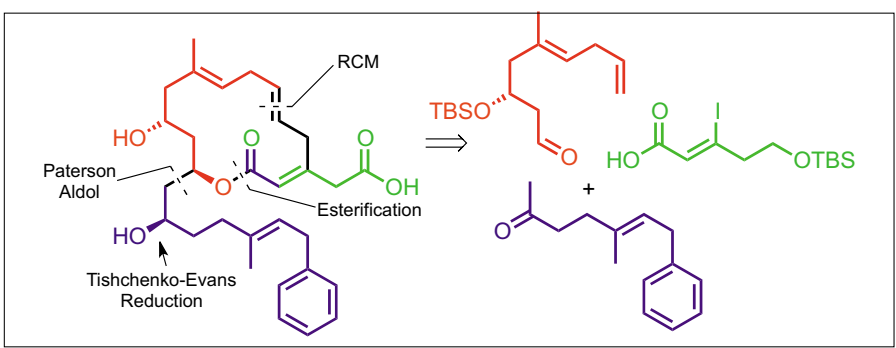

\section{Synthesis of DNA Interstrand Cross-Links Using a Photocaged Nucleobase}

S. Hentschel, J. Alzeer, T. Angelov, O. D. Schärer, and N. W. Luedtke*, Angew. Chem. Int. Ed. 2012, 51, 3466.

University of Zürich

Carmustine is a chemotherapeutic agent used to treat several types of cancer that generates DNA-DNA interstrand crosslinks. Various aspects of its biochemistry are poorly understood, as no synthetic route towards DNA containing such crosslinks was available. The authors now report the synthesis of crosslinked DNA by using a photocaged nucleobase that can be incorporated into duplex DNA. Upon irradiation, a highly reactive electrophile is formed which attacks the opposing DNA strand. This method allows for preparation of crosslinked DNA that is analogous to the product created by the drug, thus opening new possibilities to study biological pathways involved in the drug's action. The same strategy should also be applicable in other photo-controlled site-specific covalent modification reactions.

Duplex DNA containing photocaged nucleobase $0^{6}$-choroethyl-guanine $\mathrm{N}^{1}, \mathrm{O}^{6}$-ethanoguanine

Prepared by Nico Bruns, Adnan Ganic, Valentin Köhler, Fabien Monnard, Mark R. Ringenberg, and Thomas R. Ward Do you want your article to appear in this SWISS SCIENCE CONCENTRATES highlight?

Please contact concentrates@chimia.ch 